\title{
A new phenomenological formula for ground state binding energies
}

\author{
G. Gangopadhyay \\ Department of Physics, University of Calcutta \\ 92, Acharya Prafulla Chandra Road, Kolkata-700 009, India \\ email: ggphy@caluniv.ac.in
}

\begin{abstract}
A phenomenological formula based on liquid drop model has been proposed for ground state binding energies of nuclei. The effect due to bunching of single particle levels has been incorporated through a term resembling the one-body Hamiltonian. The effect of n-p interaction has been included through a function of valence nucleons. A total of 50 parameters has been used in the present calculation. The r.m.s. deviation for the binding energy values for 2140 nuclei comes out to be $0.376 \mathrm{MeV}$, and that for 1091 alpha decay energies is $0.284 \mathrm{MeV}$. The correspondence with the conventional liquid drop model is discussed.
\end{abstract}

\section{Introduction}

The charged liquid drop model of the nucleus, in combination with various modifications, has very successfully been applied to describe nuclear ground state binding energy and many other ground state properties [1]. Most of the modern global mass formulas retain the original Bethe-Weizsäcker (BW) form as basis and add certain effects such as shell correction, deformation, surface symmetry terms, etc. Fully microscopic approaches such as Skyrme Hartree Fock or Relativistic Mean Field methods also have been employed to estimate the mass values through out the periodic table. Local formulas, as the name suggests, predict the unknown mass of a nucleus by extrapolation from the known masses of neighbouring nuclei.

Fully microscopic approaches are yet to reach the accuracy achieved by microscopic-macroscopic mass formula. Skyrme Hartree Fock mass values show an r.m.s. deviation slightly less than $0.6 \mathrm{MeV}[2$. Local approaches, on the other hand, can be applied to unknown mass regions only with limited confidence. Thus, for example, the neutron rich regions, important for various astrophysical processes such as r- or s-process, are usually studied using the microscopic-macroscopic approaches.

Microscopic-macroscopic methods usually use the liquid drop model (LDM) 
as the macroscopic part of the prescription and introduce different corrections based on microscopic models. Myers and Swiatecki $[3$ included the shell effects by considering the bunching of the single particle levels. Strutinsky correction [4] takes care of the shell effects in a fully microscopic way. Finite Range Droplet Model (FRDM) [5 is an approach which, besides including Strutinsky corrections, pairing and other effects, also modifies the basic liquid drop macroscopic part of the mass formula. Another very successful approach based on a microscopic picture by Duflo and Zuker [6] yields an r.m.s. deviation of $0.373 \mathrm{MeV}$ for 2135 nuclei.

Estimation of binding energy remains a very important task in nuclear physics. For example, one of the most important inputs in the study of astrophysical reactions is the nuclear ground state binding energy. In nuclei far from the stability valley, experimental mass measurements are very scarce and likely to remain so in near future. Thus, theoretical prediction of mass remains our only guide. In the present work we present a phenomenological formula for calculation of binding energy values.

\section{Theory and results}

\subsection{The binding energy formula}

The general approach to a successful nuclear mass formula has been to assume the liquid drop model and add shell corrections to it. The liquid drop model gives a smooth curve while the shell corrections represent the effect of the single particle states. In the present work, we have employed a approach which is close to the Myers and Swiatecki prescription[3] with the shell correction being simulated using a number of parameters.

The original liquid drop model mass formula is known to give incorrect results because of the bunching of the single particle levels. To incorporate this effect, we have added a term which resembles the form of the one-body Hamiltonian. However, the single particle energies are not constants throughout the periodic table but vary with the neutron and proton numbers of the concerned nucleus. To simulate this effect, we have used a term of the form

$$
B_{\text {bunc }}=\sum_{i}^{1,2} \sum_{\alpha} \epsilon_{\alpha}^{i} \mathcal{N}^{i} n_{\alpha}^{i}
$$

where $i=1,2$ refer to the neutron or proton, respectively. The number of neutrons and protons in the nucleus are given by $\mathcal{N}^{1}(=N)$ and $\mathcal{N}^{2}(=Z)$, respectively. We assume a shell model like filling of the orbits to obtain the $n_{\alpha}^{i}$ values for different nuclei. Thus we have

$$
n_{\alpha}^{i}=\left\{\begin{array}{lll}
\mathcal{N}_{\alpha+1}^{i}-\mathcal{N}_{\alpha}^{i} & \text { for } \quad \mathcal{N}^{i}>\mathcal{N}_{\alpha+1}^{i} \\
\mathcal{N}^{i}-\mathcal{N}_{\alpha}^{i} & \text { for } \quad \mathcal{N}_{\alpha}^{i} \leq \mathcal{N}^{i} \leq \mathcal{N}_{\alpha+1}^{i} \\
0 & \text { for } \quad \mathcal{N}^{i}<\mathcal{N}_{\alpha}^{i}
\end{array}\right.
$$


Table 1: Values of various parameters for the binding energy formula obtained in the present calculation. The parameters $k_{v}, k_{s}, k_{W}$ are dimensionless while $r_{0}$ is given in $f m$. The rest of the parameters are in $\mathrm{MeV}$.

\begin{tabular}{|c|c|c|c|c|c|c|}
\hline & & $\alpha$ & $\mathcal{N}_{\alpha}^{1}$ & $\epsilon_{\alpha}^{1}$ & $\overline{\mathcal{N}_{\alpha}^{2}}$ & $\overline{\epsilon_{\alpha}^{2}}$ \\
\hline$a_{v}$ & 11.8906 & 1 & 8 & 0.1284 & 8 & 0.2027 \\
\hline$a_{\text {surf }}$ & 7.3654 & 2 & 14 & -0.0227 & 14 & 0.0284 \\
\hline$k_{v}$ & 1.6627 & 3 & 20 & 0.0651 & 20 & 0.0889 \\
\hline$k_{s}$ & -2.6383 & 4 & 24 & 0.0306 & 24 & 0.0462 \\
\hline$W$ & 35.6813 & 5 & 28 & 0.0394 & 28 & 0.0307 \\
\hline$k_{W}$ & 8.3735 & 6 & 32 & 0.0221 & 40 & 0.0191 \\
\hline$a_{s}$ & $3.1619 \times 10^{-8}$ & 7 & 40 & 0.0102 & 44 & 0.0256 \\
\hline$r_{0}$ & 1.0625 & 8 & 44 & 0.0248 & 50 & 0.0120 \\
\hline$a_{c}^{\prime}$ & $-6.8171 \times 10^{-3}$ & 9 & 50 & 0.0089 & 64 & 0.0106 \\
\hline$a_{n}$ & -1.0645 & 10 & 60 & 0.0138 & 74 & 0.0079 \\
\hline$a_{p}$ & -0.9508 & 11 & 64 & 0.0093 & 80 & 0.0050 \\
\hline$a_{n p}^{2}$ & $3.3594 \times 10^{-2}$ & 12 & 76 & 0.0118 & 82 & -0.0001 \\
\hline$a_{n p}^{3}$ & $-3.2543 \times 10^{-4}$ & 13 & 82 & 0.0031 & 88 & 0.0013 \\
\hline$a_{\delta}$ & 4.7686 & 14 & 88 & 0.0079 & 96 & -0.0022 \\
\hline \multirow[t]{4}{*}{$a_{\delta}^{\prime}$} & -1.4162 & 15 & 96 & 0.0074 & & \\
\hline & & 16 & 122 & 0.0041 & & \\
\hline & & 17 & 126 & 0.0043 & & \\
\hline & & 18 & 132 & 0.0078 & & \\
\hline
\end{tabular}

The different $\mathcal{N}_{\alpha}^{i}$ values are estimated in the following way. While fitting the different parameters in the formula for binding energy, we have checked the errors at different neutron and proton numbers i.e. for different nuclei. Whenever, the errors tend to be large at a particular $N$ or $Z$ value, we assume that the single particle structure has changed at that particular number and new energy level has been occupied. Thus we have assumed $\epsilon_{\alpha}$ values at different neutron or proton numbers $\left(\mathcal{N}_{\alpha}^{i}\right)$ as given in the last five columns of table 1. This particular form remains an ad hoc assumption in our work; yet we find that it can successfully explain the binding energy values.

Some of the $\mathcal{N}_{\alpha}^{i}$ values are easy to understand. The conventional magic numbers and semi-magic numbers do occur in the scheme naturally. On the other hand, some other values may be associated with subshell closures. For example, $\mathcal{N}_{\alpha}=14,32,64$ and 76 (some in the case of neutrons only) agree with the $1 d_{5 / 2}, 1 p_{3 / 2}, 2 d_{5 / 2}$ and $1 h_{11 / 2}$ subshell closures. Similarly, $N=88$ may be associated with a deformed shell closure. Weaker deformed shell closure observed at $N=98$ and $Z=100$ are also very close to the value $N_{\alpha}=96$ that we have obtained. The readers are referred to Refs. [7, 8, 9] for more details 
on shell closures and deformed magic numbers. We should also mention that Schwierz et al. [10 in a study on Woods Saxon potential have found indications of smaller gaps at $N, Z=14$ and 32 in the light mass region. Sorlin et al.[11] have pointed out the evidence for subshell closures at 14, 32 and 64 .

We have also introduced modifications in a few $\epsilon$ values and the $\mathcal{N}_{\alpha}^{i}$ values at which they occur for a number of nuclei. This is done by again looking at the deviations and checking whether there is any systematic pattern. In nuclei with $Z \leq 22$ and $N \geq 29$, we find that a better result is obtained if we change the $\epsilon_{2}^{2}$ and $\epsilon_{3}^{2}$ values, i.e. for proton numbers corresponding to 14 and 20, respectively. Similarly, in nuclei with $Z \leq 35$ and $N \geq 46$, we find that a better result is obtained if we change the value of the parameter $\epsilon_{8}^{1}$ (corresponding to neutron number 44) and assume that the next change in single particle level does not correspond to $N=50$. Interestingly, this may correspond to the possible disappearance of the magic gap at $N=50$ in nuclei with $Z \leq 35$. A similar situation appears in nuclei with $Z \leq 42$ where the bunching at $N=60$ seems to disappear. The fact that all the above changes occur in neutron rich nuclei is a signature of rearrangement of single particle levels expected in such regions.

The Binding energy formula used in the present work is given by

$$
\begin{array}{r}
B . E .(N, Z)=B_{L D M}+B_{\text {bunc }}+B_{n p}+B_{e l} \\
B_{L D M}=a_{v}\left(1-k_{v} I^{2}\right) A-a_{\text {surf }}\left(1-k_{s} I^{2}\right) A^{2 / 3}-B_{\text {sym }}-B_{\text {Coul }} \\
-B_{\text {pair }} \\
B_{\text {sym }}=W|I|\left(1-k_{W}|I|\right)+a_{s}(N-Z)^{5} \\
B_{\text {Coul }}=\frac{3}{5} \frac{Z(Z-1) e^{2}}{r_{0} A^{1 / 3}}+a_{c}^{\prime}\left(\frac{Z^{2}}{A}\right)^{5 / 2} \\
B_{\text {pair }}=a_{\delta}\left(\frac{\delta_{n}}{N^{1 / 3}}+\frac{\delta_{p}}{Z^{1 / 3}}\right)+a_{\delta}^{\prime} \frac{\delta_{n} \delta_{p}}{A^{1 / 3}} \\
B_{n p}=a_{n} N_{p}+a_{p} N_{n}+a_{n p}^{2}\left(N_{p}+N_{n}\right)^{2}+a_{n p}^{3}\left(N_{p}+N_{n}\right)^{3}
\end{array}
$$

Here, $\delta_{n(p)}$ is 0 or 1 , according as $N(Z)$ is even or odd. We write $I=$ $(N-Z) / A$. The expression for $B_{b u n c}$ is already given in eqn (1). In $B_{n p}, N_{p}$ and $N_{n}$ refers to the number of valence protons and neutrons. The electronic binding energy is estimated by the empirical relation

$$
B_{e l}(\mathrm{MeV})=1.44381 \times 10^{-5} Z^{2.39}+1.55468 \times 10^{-12} Z^{5.35}
$$

The liquid drop part $B_{L D M}$ contains a number of correction terms along with the original BW prescription. As $B_{\text {bunc }}$ contain a large number of $\epsilon_{\alpha}^{i}$ 's, we have a large number of parameters, viz. fifty in number. If we also consider that the nucleon numbers for the bunching of the levels as parameters, the number of increases substantially. One obvious problem in our approach is that the nucleon numbers used in the bunching part of the formula do not come naturally but have to be introduced from experimental mass values. Only microscopic calculations can possibly supplement experimental inputs in this regard. 
The bunching term already includes a significant part of the total energy. Thus we expect a major modification of the liquid drop model coefficients as well as the functional form of some of the smaller correction terms. We have included some different functional forms for the latter terms as described below.

The volume and the surface terms as well the contribution of symmetry energy to them are retained in the LDM part of the formula. However, the parameters are expected to be substantially modified in the present approach. Another major modification that we find is in the symmetry energy term $B_{\text {sym }}$. The term proportional to $|I|$ is the Wigner term that was introduced from the symmetry argument. We have introduced two additional terms in the symmetry as is clear from the expression. FRDM suggests that the charge asymmetry contribution should be large in heavier nuclei and uses a term proportional to $(N-Z)$. We find that a term $(N-Z)^{5}$ fits the data better in our case.

The Coulomb energy is represented as a sum of two terms. The first one is the usual Coulomb term for a charged liquid drop. For the second term, we have used a functional of $Z^{2} / A$. The usual Coulomb contributions include an exchange term of the form $Z^{4 / 3} / A^{1 / 3}$, proton form factor correction of the form $Z^{2} / A$. The FRDM also has contributions from volume and surface rearrangement terms which depend on $Z$. Lunney et al. [1] have also used a term proportional to $Z^{4} / A^{2}$ in the macroscopic part of the energy functional related to surface stiffness. We have included a term of the form $\left(Z^{2} / A\right)^{5 / 2}$ as a single term representing the Coulomb corrections. We should mention that a nearly comparable fit can be achieved using a form $\left(Z^{2} / A\right)^{2}$ also.

Another addition is the $B_{n p}$ term which is a part of the microscopic energy. It has been demonstrated[12, 13, that the number of valence neutrons and protons show a decidedly strong correlation with n-p interaction and thus with deformation. We have taken a form of the type shown in eqn (7). The number of valence particles or holes has been calculated from the nearest major shell, i.e. $8,20,28,5082,126$ and 184 . We have also tried to fit the data using 172 as the last major shell. We find that the fit is slightly worse in this case. However, in keeping with the expectation that the shell closures are less pronounced in the SHE region, the binding energy data is not really sufficient to conclude whether the next major closure is at $N=184$ or 172 . On the other hand, as explained later, we have also calculated the $\mathrm{Q}$-value in $\alpha$-decay where the former value gives decidedly better results and we have used it in our calculation. The dependence of nuclear mass on valence nucleons has already been studied by Mendoja-Temis et al. [14 in a different form.

The pairing energy has been shifted so that the value for $B_{\text {pair }}$ is zero in even-even nuclei. We have also assumed a $A^{-1 / 3}$ dependence for the n-p pairing term.

\section{$2.2 \quad$ Parameters}

The justification of using such a large number of parameters can only be through a good and simple prediction. One advantage of using this formula is that one may use it directly without microscopic correction as the bunching due to shell 


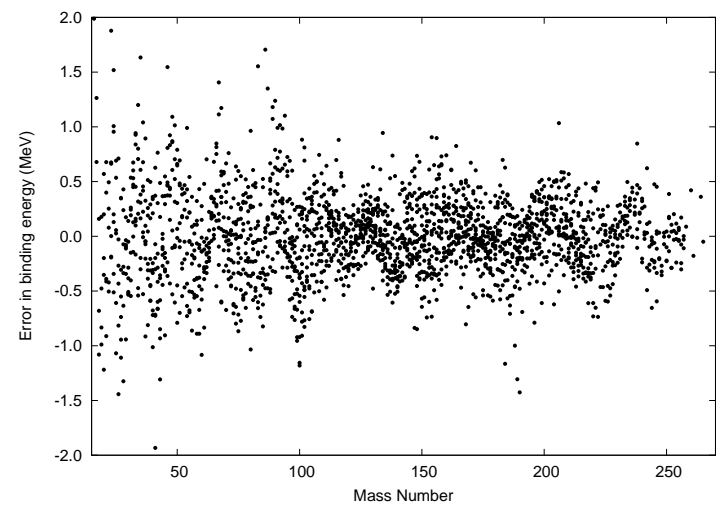

Figure 1: Deviations of binding energy for 2140 nuclei with $N, Z \geq 8$ from the predictions of the present formula.

effects are included in the $B_{\text {bunc }}$ term. We have fitted the experimental masses in two steps as explained below.

In the first step we have included all the experimental mass values in nuclei with $N, Z \geq 8$ from the mass table by Audi et al.[15]. A total of 2140 nuclei have been used in the fitting procedure yielding an r.m.s. deviation of $0.378 \mathrm{MeV}$. More importantly, there are only 39 nuclei which show deviation larger than 1 $\mathrm{MeV}$ and none more than $2 \mathrm{MeV}$. In nuclei with mass more than 100, only 4 nuclei show a deviation larger than $1 \mathrm{MeV}$ and none more than $1.5 \mathrm{MeV}$. We would like to note that if the modifications in the three $\epsilon_{i}$ values, as explained in the previous subsection, are not introduced for neutron rich nuclei, the r.m.s. deviation becomes $0.400 \mathrm{MeV}$. In this case we also assume that for the nuclei with $N<22$ and 34, the bunching at $N=28$ and 44, respectively, disappears.

It should be interesting to compare the results for some measurements performed later than 2003 and not included in the mass table 15 mentioned above. The masses of a number of nuclei have been measured either for the first time or with increased precision in the last few years. We have looked for measurements which are completely new or which substantially differ from previous values at least in some of the nuclei studied. All the measurements from such references have been included for completeness. Refs. [16 ${ }^{-} 31$ have been selected for inclusion. These measurements have been divided in two groups. The first group contains those nuclei for which experimental values were available in Audi et al. [15] but later measurements have modified them. There are 134 nuclei in this group. In the second group, there are 43 nuclei whose experimental binding energy values have been measured later and were not tabulated in Audi et al.[15. For both the groups taken together, the r.m.s. error is $0.438 \mathrm{MeV}$. For the second group alone, the r.m.s. deviation is $0.517 \mathrm{MeV}$.

In the second step, we have replaced the binding energy values of the nuclei belonging to the first group with the improved binding energy measurements obtained later wherever available. A fitting procedure for 2140 nuclei now show 


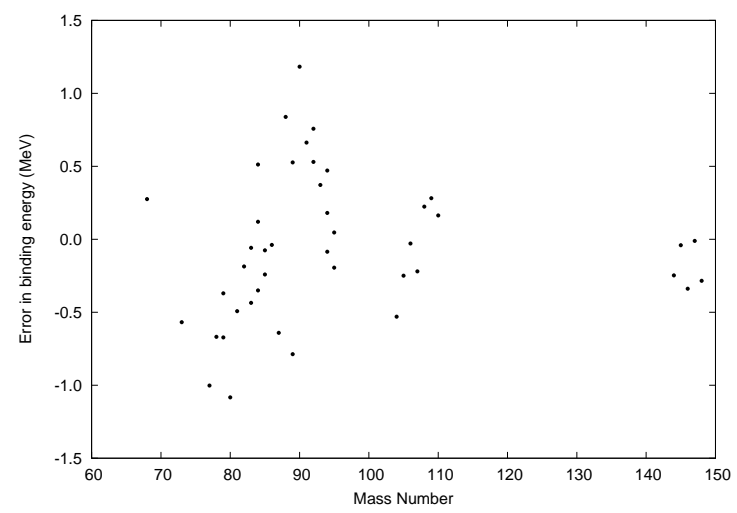

Figure 2: Deviations of binding energy for 43 nuclei whose binding energy values have recently measured from the predictions of the present formula.

Table 2: Deviation in some global mass formulas

\begin{tabular}{lccc}
\hline & No. of nuclei & $\begin{array}{c}\text { No. of } \\
\text { parameters }\end{array}$ & $\begin{array}{c}\text { r.m.s. } \\
\text { deviation } \\
(\mathrm{MeV})\end{array}$ \\
\hline Myers-Swiatecki[35] & 1312 & 50 & 0.663 \\
Skyrme-HFB[2] & 2149 & 24 & 0.581 \\
Duflo-Zuker[6] & 2135 & 28 & 0.373 \\
FRDM[5] & 2135 & 33 & 0.676 \\
Present & 2140 & 50 & 0.376 \\
\hline
\end{tabular}

an r.m.s. deviation of $0.376 \mathrm{MeV}$. The quality of fit is as good as obtained in the first step. The new parameters are very close to the fitted values in the first case. The new fitted parameters also describe the values in the second group slightly better, the r.m.s. deviation being $0.494 \mathrm{MeV}$. Fig. 1 shows the deviation from the prediction of the (2) formula for the 2140 nuclei while Fig. 2 is the corresponding figure for the 43 new measurements. The parameters are given in table 1.

In table 2 we compare our results with some other global calculations. It is clear that our calculation uses more free parameters. Besides, a number of parameters in the other approaches are predetermined from considerations other than mass measurements. However, as already noted, the advantage of the present formula is that it is an analytic expression, and not dependent on input from any other calculation.

To check the agreement of our results with other predictions, we have chosen two isotope chains, $Z=50$ and $Z=110$. The former has been chosen because experimental binding energy values for a long chain of isotopes, from $N=50$ to 

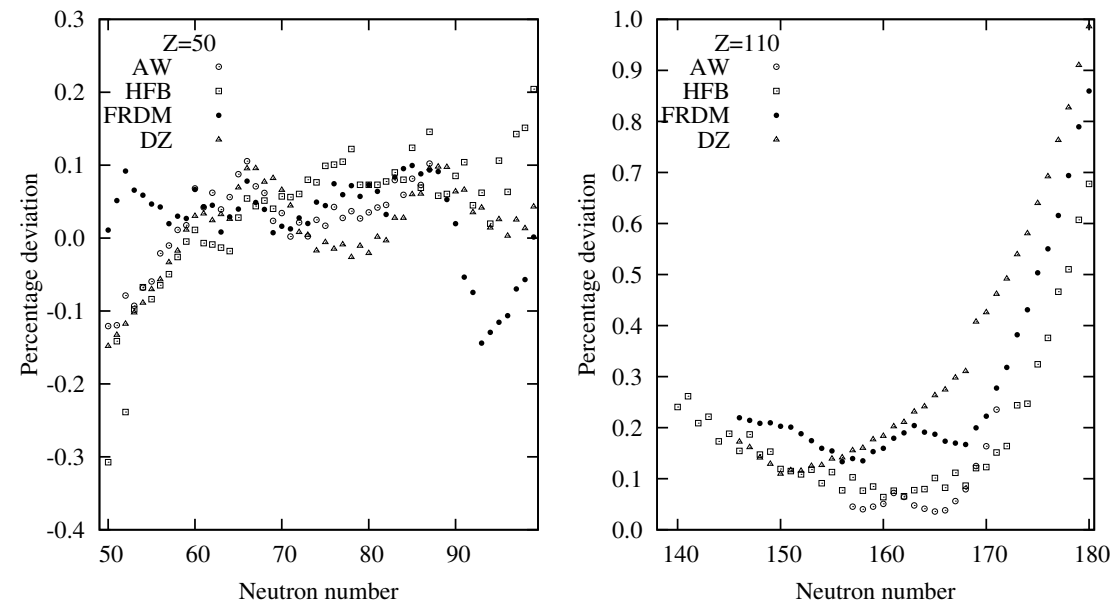

Figure 3: Differences of the present calculation from experimental values and other prescriptions for $Z=50$ and $Z=110$ nuclei. See text for details.

$N=84$, are available. The other has been chosen to compare the predictions in a region where no experimental data is available. The percentage deviation values for binding energy are plotted in Fig. 3. The legends AW correspond to the experimental or estimated values from the mass table [15. The legends HFB, FRDM and DZ refer to the calculations according to the Skyrme Hartree Fock Bogoliubov calculation 2, FRDM calculation [5 and Duflo Zuker ten parameter formula $[6$.

The figure indicates that the present formula agree well for the $\mathrm{Z}=50$ isotope chain. However, the right panel show that there is a systematic deviation between the present result and the predictions of HFB, FRDM or Duflo-Zuker calculations for $Z=110$ isotopes. The present model systematically predicts less binding energy for all the nuclei in the chain. More importantly, beyond $N=172$, the deviations with all the three theoretical calculations start to rise. When experimental mass values for these nuclei become available, it will be clear whether the present prescription needs to be modified. It needs to be remembered that the largest values that have been used in the fitting procedure are $N=159$ and $Z=108$, as available in Audi et al. [15].

As a further check on the present prescription, we have calculated the alpha decay energies in 1091 decays obtained from Audi et al. [15] augmented by the latest results available in the National Nuclear Data Center website 32. The 


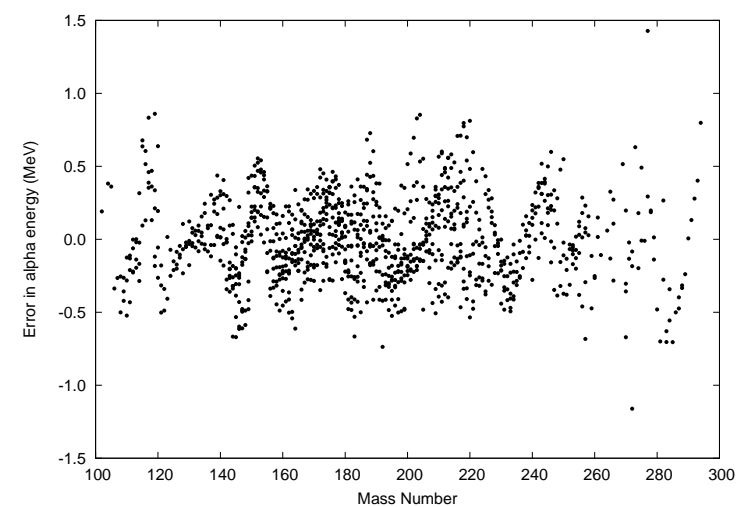

Figure 4: Error in alpha decay energy for 1091 alpha decays

r.m.s. deviation for all these decay energies is $0.284 \mathrm{MeV}$, a value consistent with the accuracy in the prediction of binding energy in the present work. The deviations in alpha decay energies are plotted in Fig. 4.

It is possible that beyond the highest nucleon numbers with known experimental binding energy values, i.e. $N=159$ and $Z=108$, there are other nucleon numbers where we may have to introduce additional bunching. We have looked at the alpha decay energies for nuclei with larger $N$ or $Z$ values and have tried to fit with different $\epsilon$ parameters at different $N$ or $Z$ numbers. A least square fitting did not yield a substantial improvement in the alpha decay energies. However, as the number of observed decays in this region is not large, it is not possible to conclude positively about possible change in bunching beyond the above $N$ or $Z$ values. We have already noted that alpha decay Q-values indicate that $N=184$ is a better closed core than 172 .

\subsection{Correspondence with the liquid drop model}

We see that the usual liquid drop model parameters are modified to a large extent in the present calculation. It is difficult to compare the standard values of parameters with the specific form in (1). Parts of all the conventional liquid drop model terms, such as the volume, surface, symmetry and Coulomb energies, are included in the bunching part of the formula. Thus the values of the different parameters in the present work differ substantially from the values obtained from their counterparts in conventional calculations. Even a parameter like $r_{0}$, which seems to take a value in conformity with other results, is actually very much dependent on the form of the functional chosen for the second term in $B_{\text {Coul }}$.

It is possible to visualize our results in the following way. In $N=Z$ nuclei, there is no contribution from symmetry energy. We would like to interpret the difference in contribution from the proton and the neutrons to $B_{\text {bunc }}$ in such nuclei as essentially a part of the Coulomb energy in the conventional 
LDM. Thus we have written the Coulomb energy in $N=Z$ nuclei, neglecting the difference between the neutron and proton energies in $B_{n p}$, as $E_{C o u l}=$ $B_{\text {Coul }}+B_{\text {bunc }}^{p}-B_{\text {bunc }}^{n}$. This quantity we have calculated for $N=Z$ nuclei up to 108 from the proposed binding energy formula using the parameters deduced above and have plotted it with a simple conventional form consisting of a direct term and a diffuseness correction, i.e.

$$
\frac{3}{5} \frac{e^{2} Z^{2}}{r_{0} A^{1 / 3}}+f_{p} \frac{Z^{2}}{A}
$$

Royer and Rousseau[33] pointed out that the Coulomb exchange term is unnecessary if the diffuseness correction is included. Fitting $E_{C o u l}$ with the above expression, we obtain $r_{0}=1.2114 \mathrm{fm}$ and $f_{p}=1.0395 \mathrm{MeV}$ which are within the range of usual values 34 . The Coulomb energy $E_{C o u l}$ is better described using a functional of the form

$$
\frac{3}{5} \frac{e^{2} Z^{2}}{r_{0} A^{1 / 3}}+f_{p} \frac{Z^{2}}{A}+a_{c h} \frac{Z^{4}}{A^{2}}
$$

In even-even $N=Z$ nuclei, it is possible to extract the volume and surface term coefficients from the mass formula. The sum of volume and surface terms is obtained by adding $E_{\text {Coul }}$ to the total B.E. from eqn (2) taking the microscopic corrections into account and fitted with a functional of the form

$$
\alpha_{v} A-\alpha_{s} A^{2 / 3}
$$

The values of the coefficients $\alpha_{v}$ and $\alpha_{s}$ have been obtained in this way. We have considered only nuclei for which the microscopic correction terms from [5] are available.

With the equivalent volume, surface and Coulomb energies given by the prescriptions (10) and (11), it is possible to estimate the symmetry energy contained in the values obtained from eqn (2). We have calculated the symmetry energy for all the even even nuclei among the 2140 which we have fitted originally taking into account the microscopic corrections from Möller et al. [5. There are various possible expressions for the symmetry energy in LDM and we have taken a functional of the form

$$
-a_{v} k_{v} I^{2} A+a_{s} k_{s} I^{2} A^{2 / 3}-W|I|+c_{s}(N-Z)
$$

the last term being used in the FRDM. The different parameters have been estimated by least square fitting.

The interpretation of pairing energy terms for odd- $\mathrm{Z}$ or odd- $\mathrm{N}$ terms are straightforward. The coefficient of the $1 / \sqrt{N(Z)}$ term is $4.77 \mathrm{MeV}$, a value very close to the accepted value of $4.8 \mathrm{MeV}$. The $\mathrm{n}$-p pairing term is usually taken in a different form from the present work and cannot be directly compared. Its effect is of course small.

Following the procedure described above, we get the following expression for binding energy in terms of the deduced parameters:

$$
B(\mathrm{MeV})=15.2893\left(1-1.8735 I^{2}\right) A-16.4662\left(1-2.4595 I^{2}\right) A^{2 / 3}
$$




$$
\begin{gathered}
-\frac{3}{5} \frac{e^{2} Z^{2}}{1.3662 A^{1 / 3}}+0.3639 \frac{Z^{2}}{A}-0.0421 \frac{Z^{4}}{A^{2}} \\
-28.0438|I|-0.4857(N-Z)-B_{\text {pair }}-E_{\text {shell }}
\end{gathered}
$$

where $E_{\text {shell }}$ values are the microscopic corrections from Möller et al. $[\mathbf{5}$. It is seen that the coefficients in the above formula are within the accepted ranges for their counterparts in the liquid drop model. We note that the parameters in the above expression have been obtained fitting, not the experimental values, but the values predicted by eqn (2). Using the above formula, we find that the r.m.s. deviation from experimental values is obtained as $0.969 \mathrm{MeV}$ for the nuclei in Audi et al.15. Thus, we may conclude that the present prescription is equivalent to a conventional LDM formula which takes shell corrections into account.

\section{Summary}

A new phenomenological formula has been proposed for ground state binding energies of nuclei. Two additional components have been included in the proposed formula in addition to the conventional liquid drop model. The contribution of the bunching of single particle levels has been included through a term resembling the one-body Hamiltonian. No additional shell correction from any external calculation has been considered. The effect of n-p interaction and deformation has been included through a function of the number of valence nucleons. A total of 50 parameters has been used in the present calculation. The r.m.s. deviation in the ground state binding energy for 2140 nuclei comes out to be $0.376 \mathrm{MeV}$. As an additional check on the formula, decay energies for 1091 alpha decaying nuclei has been calculated which yields an r.m.s. deviation of $0.284 \mathrm{MeV}$. As the bunching term incorporates a large part of the conventional liquid drop model terms, the parameters in the present work differ substantially form their standard values. The present formula may be reduced to the form of a conventional liquid drop model with parameters close to standard values.

\section{Acknowledgment}

This work is carried out with financial assistance of the UGC sponsored DRS Programme of the Department of Physics of the University of Calcutta. The author would like to thank T.K. Das for reading the manuscript and making helpful suggestions.

\section{References}

[1] See D. Lunney, J.M. Pearson and C. Thibault 2003 Rev. Mod. Phys. 75 (2003) 1021 for a comprehensive review of the various theoretical approaches.

[2] S. Goriely, J. Chamel and J.M. Pearson, Phys. Rev. Lett. 102 (2009) 152503. 
[3] W.D. Myers and W.J. Swiatecki, Nucl. Phys. 81 (1966) 1.

[4] V.M. Strutinsky Nucl. Phys. A95 (1967) 420.

[5] See P. Möller, J.R. Nix, W.D. Myers and W.J. Swiatecki, At. Data. Nucl. Data. Tables 59 (1995) 185 for details.

[6] J. Duflo and A.P. Zuker, Phys. Rev. C52 (1995) R23; Phys. Rev. C59 (1999) $\mathrm{R} 2347$.

[7] A. Bohr and B. Mottelson, Nuclear Structure, Vol I, Single-Particle Motion, W. A. Benjamin (1969).

[8] A. Bohr and B. Mottelson, Nuclear Structure, Vol II, Nuclear Deformations, W. A. Benjamin (1975).

[9] J. Maruhn and W. Greiner. Z. Phys. 251 (1972) 431.

[10] N. Schwierz, I Widenhöver and A. Volys, arXiv:0709.3525 (2007).

[11] O. Sorlin and M.-G. Porquet, Prog. in Part. Nucl. Phys. 61 (2008) 602.

[12] R.F. Casten, Phys. Rev. Lett. 54 (1985) 1991; R.F. Casten and N.V. Zamfir, Phys. Rev. Lett. 70 (1993) 402.

[13] M. Bhattacharya and G. Gangopadhyay, Phys. Lett. B672 (2009) 182; G. Gangopadhyay, J. Phys. G: Nucl. Part. Phys. 37 (2010) 015108.

[14] J. Mendoja-Temis et al., Nucl. Phys. A799 (2008) 84.

[15] G. Audi, A.H. Wapstra, and C. Thibault, Nucl. Phys. A729 (2003) 337.

[16] G. Sikler et al., Nucl. Phys. A763 (2005) 45.

[17] U. Hager et al., Phys. Rev. Lett. 96 (2006) 042504.

[18] A. Kankainen et al., Eur. Phys. J. A29 (2006) 271.

[19] S. Rahaman et al., Eur. Phys. J. A32 (2007) 87.

[20] S. Rahaman et al., Eur. Phys. J. A34 (2007) 5.

[21] U. Hager et al., Nucl. Phys. A793 (2007) 20.

[22] P. Schury et al., Phys. Rev. C 75 (2007) 055801.

[23] U. Hager et al., Phys. Rev. C 75 (2007) 064302.

[24] P. Delahaye et al., Phys. Rev. C 74 (2006) 034331.

[25] M.B. Tsang et al., Phys. Rev. C 76 (2007) 041302(R).

[26] M. Dworschak et al., Phys. Rev. Lett. 100 (2008) 072501. 
[27] C. Rauth et al., Phys. Rev. Lett. 100 (2008) 012501.

[28] S. Baruah et al., Phys. Rev. Lett. 101 (2008) 262501.

[29] J. Hakala J et al., Phys. Rev. Lett. 101 (2008) 052502.

[30] M.B. Gomez-Hornillo et al., Phys. Rev. C 78 (2008) 014311.

[31] C. Weber et al., Phys. Rev. C 78 (2008) 054310.

[32] http://www.nndc.bnl.gov

[33] G. Royer and Rousseau, Eur. Phys. J. A (2009) 541.

[34] G. Royer and C. Gautier, Phys. Rev. C 73 (2006) 067302; G. Royer, Nucl. Phys. A807 (2008) 105.

[35] H. v. Groote, E.R. Hilf and K. Takahashi, At. Data. Nucl. Data. Tables 17 (1976) 418. 